Proceedings

\title{
Anxiolytic-Like Effects of Lupinus angustifolious Protein Hydrolysates in Alzheimer Model Mice ${ }^{+}$
}

\author{
Guillermo Santos-Sánchez 1,2,‡, Eduardo Ponce-España 1,2,, Ivan Cruz-Chamorro 1,2, Juan Carlos López ${ }^{3}$, \\ Ana Isabel Álvarez-López ${ }^{1,2}$, Justo Pedroche ${ }^{4}$, María Carmen Millán-Linares ${ }^{5}$, Francisco Millán ${ }^{4}$, \\ Patricia Judith Lardone 1,2, Ignacio Bejarano ${ }^{1,2}$, Juan Miguel Guerrero 1,2,6 and Antonio Carrillo-Vico 1,2,*
}

Citation: Santos-Sánchez, G.; Ponce-España, E.; Cruz-Chamorro, I.; López, J.C.; Álvarez-López, A.I.; Pedroche, J.; Millán-Linares, M.C.; Millán, F.; Lardone, P.J.; Bejarano, I.; et al. Anxiolytic-like Effects of

Lupinus angustifolious Protein

Hydrolysates in Alzheimer Model Mice. Proceedings 2021, 70, 41. https:/ doi.org/10.3390/foods_2020-07685

Published: 9 November 2020

Publisher's Note: MDPI stays neutral with regard to jurisdictional claims in published maps and institutional affiliations.

Copyright: (C) 2020 by the authors. Licensee MDPI, Basel, Switzerland. This article is an open access article distributed under the terms and conditions of the Creative Commons Attribution (CC BY) license (http://creativecommons.org/licenses/by/4.0/).
1 Instituto de Biomedicina de Sevilla, IBiS (Universidad de Sevilla, HUVR, Junta de Andalucía, CSIC), 41013 Seville, Spain; gsantos-ibis@us.es (G.S.-S.); eponce-ibis@us.es (E.P.-E.); icruz-ibis@us.es (I.C.-C.); aialvarez-ibis@us.es (A.I.Á.-L.); plardone@us.es (P.J.L.); ibejarano@us.es (I.B.); guerrero@us.es (J.M.G.)

2 Departamento de Bioquímica Médica y Biología Molecular e Inmunología, Universidad de Sevilla, 41009 Seville, Spain

3 Departamento de Psicología Experimental, Universidad de Sevilla, 41009 Seville, Spain; jclopez@us.es

4 Department of Food \& Health, Instituto de la grasa, CSIC, Ctra, Utrera Km 1, 41013 Seville, Spain; jjavier@cica.es (J.P.); frmillan@cica.es (F.M.)

5 Cell Biology Unit, Instituto de la grasa, CSIC, Ctra, Utrera Km 1, 41013 Seville, Spain; mcmillan@ig.csic.es

6 Departamento de Bioquímica Clínica, Unidad de Gestión de Laboratorios, Hospital Universitario Virgen del Rocío, 41013 Seville, Spain

* Correspondence: vico@us.es; Tel.: +34-95-592-3106; Fax: +34-95-490-7048

† Presented at the 1st International Electronic Conference on Food Science and Functional Foods, 10-25 November 2020; Available online: https://foods_2020.sciforum.net/.

$\ddagger$ To be considered as equal first author.

\begin{abstract}
Alzheimer's disease (AD), which is characterized by a devastating and progressive loss of memory, is the principal neurodegenerative disease in the elderly population worldwide. As a consequence, $\mathrm{AD}$ patients present neuropsychiatric symptoms, such as anxiety, causing sleeping difficulty, irritability, agitation, or aggressiveness. Previous studies have demonstrated that a high-fat diet, in addition to exacerbating AD, aggravates anxiety. We have demonstrated that Lupinus angustifolius protein hydrolysates (LPHs) have anti-inflammatory and antioxidant effects, key factors for AD and anxiety. Thus, this study aimed to evaluate the potential effects of LPHs on spatial memory and anxiety of a preclinical model of $\mathrm{AD}$. ApoE $\mathrm{E}^{-/-}$mice fed with a western diet were intragastrically treated with LPHs (or a vehicle) for 14 weeks. Spatial memory and anxiety were then assessed through the Morris water maze and elevated plus maze, respectively. The results did not show significant differences in spatial memory between groups. However, a significant increase $(p$ $<0.05$ ) in time in open arms, center time, the number of crossings, and a reduction of anxiety behavior were observed in LPH-treated mice. This is the first study showing that an LPH treatment causes anxiolytic effects, pointing to LPHs as a potential component of future nutritional therapies.
\end{abstract}

Keywords: Alzheimer's; anxiety; lupine protein hydrolysates; ApoE ${ }^{-/}$; functional food ingredient

\section{Introduction}

Alzheimer's disease (AD) is a neurodegenerative disorder characterized by quick progressive cognitive dysfunction, loss of memory, and behavior impairment. AD is the world's biggest cause of dementia, affecting more than 40 million people [1]. Besides principal symptoms, AD produces a variety of neuropsychiatric effects, such as anxiety, which generates sleep disturbances, irritability, agitation, or aggressiveness [2]. Although the pathophysiological mechanisms of AD have not yet been fully described, diet westernization is described as one of the main causes of the increase in risk factors of AD development, such as cerebrovascular diseases, diabetes, hypertension, obesity, and 
dyslipidemia [3,4]. In this context, previous studies have demonstrated that a high-fat diet, in addition to exacerbating $\mathrm{AD}$, aggravates anxiety [5]. The relationship between diet and $\mathrm{AD}$ has generated a search for nutritional treatments that ameliorate the symptoms and consequences of AD. Hence, the beneficial effects of vegetable peptides from soy [6] on improving memory activity and anxiety have been demonstrated.

We have previously demonstrated that Lupinus angustifolius protein hydrolysates (LPHs) reduce inflammation and oxidative stress [7], key risk factors for AD and anxiety. Thus, this study aimed to evaluate the potential effects of LPHs on spatial memory and anxiety of a preclinical model of $\mathrm{AD}$, the $\mathrm{ApoE}^{-/-}$mice.

\section{Materials and Methods}

\section{1. $\mathrm{LPHs}$ Preparation}

LPHs were synthesized as described previously [7].

\subsection{Animals and Experimental Design}

The experimental design is shown in Figure 1. Twelve male ApoE-/- (B6.129P2-Apo-

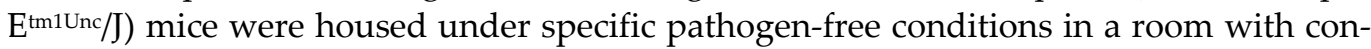
trolled temperature $\left(22 \pm 2{ }^{\circ} \mathrm{C}\right)$, humidity $(<55 \%)$, and $12 \mathrm{~h} \mathrm{light/dark} \mathrm{cycle.} \mathrm{Animals} \mathrm{had}$ free access to water and food. Animals were initially classified in two groups: mice that were fed with a standard diet (SD, $n=4)$ or a western diet (WD, $n=8)$ from the Special Diets Production Section of the University of Granada (Granada, Spain). Six-week-old mice from the WD group were randomly divided into two groups and treated intragastrically with LPHs $(100 \mathrm{mg} / \mathrm{kg}$ ) or a vehicle for 14 weeks, respectively. Individual body weight was measured and recorded weekly. Behavioral tests were performed at the Laboratory of Animal Behavior \& Neuroscience (Facultad de Psicología, Seville, Spain). Before performing the behavioral tests, animals were conditioned to these installations for a week. After the experiments, mice were euthanized with an intraperitoneal injection of sodium thiopental $(50 \mathrm{mg} / \mathrm{Kg}$, B. Braun Medical SA, Barcelona, Spain). The experimental procedures were approved by the Virgen Macarena-Virgen del Rocío University Hospital ethical committee (reference number 21/06/2016/105) and conducted under Spanish legislation and the EU Directive 2010/63/EU for animal experiments.

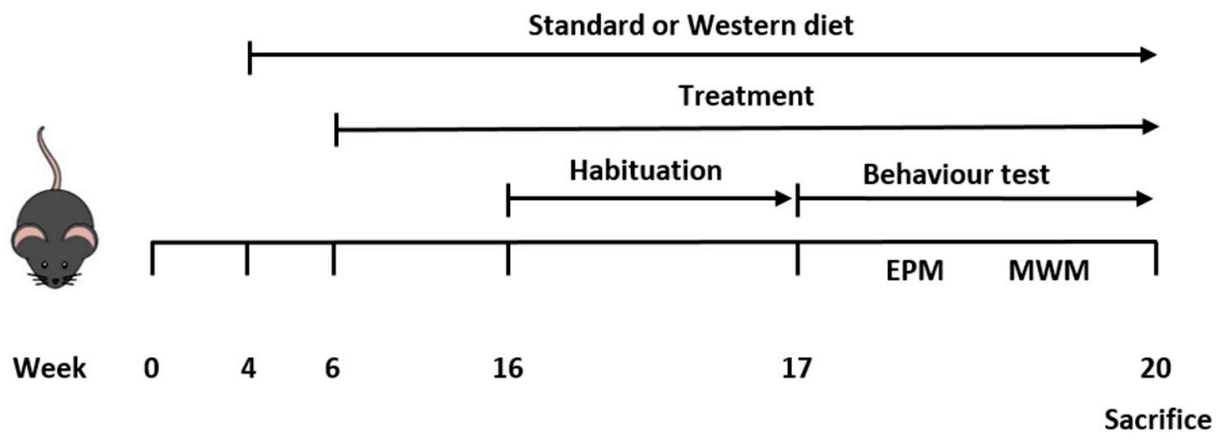

Figure 1. Experimental design and timeline. Schematic diagram of the experimental design of the study showing the mice groups, diet, and intervention. EPM, elevated plus maze; MWM, Morris water maze.

\subsection{Behavioral Test}

\subsubsection{Elevated Plus Maze}

Anxiety-like behavior was evaluated using the elevated plus maze (EPM) test at 17 weeks of age, after 12 weeks of consumption of SD, WD, or WD + LPHs. The test was performed as previously described [8]. All of the sessions were recorded using a camera 
located over the maze. Other anxiety-related behaviors, such as head dips and rears, were considered. Afterwards, the recording was processed using the plugin Animal Tracker for ImageJ software v1.52a and the time spent in the arms and center of the maze were measured.

\subsubsection{Morris Water Maze}

The Morris water maze (MWM) was performed at 19 weeks to study the processes of spatial memory and learning. Experimental procedures were performed as described by Janseen et al. [9]. All sessions were recorded with a video tracking system overlooking the pool from above. The test started at 11:30 a.m. during the light phase of the light/dark cycle, and nobody stayed in the room during the test. The latency time, distance traveled, and the time spent in each quadrant were analyzed using the plugin Animal Tracker for ImageJ software.

\subsection{Statistical Analysis}

All results were presented as mean \pm SEM using GraphPad Prism 7 software (San Diego, CA, USA). Statistical differences were evaluated using the non-parametric MannWhitney test, and $p<0.05$ was considered statistically significant. The analysis was performed using SPSS ${ }^{\circledR}$ software v24 (IBM Corporation, Armonk, NY, USA)

\section{Results and Discussion}

\subsection{LPHs Treatment Does Not Change Body Weight}

As shown in Table 1, no differences were observed in the baseline body weight (BBW), final body weight (FBW), and body weight gain (BWG) between groups.

Table 1. Body weight parameters.

\begin{tabular}{cccc}
\hline Parameter & SD & Ctrl & LPHs \\
\hline BBW $(\mathrm{g})$ & $20.35 \pm 0.41$ & $20.98 \pm 0.36$ & $20.88 \pm 0.49$ \\
FBW $(\mathrm{g})$ & $26.20 \pm 0.87$ & $26.50 \pm 0.54$ & $27.15 \pm 0.69$ \\
BWG $(\mathrm{g})$ & $5.85 \pm 1.18$ & $5.53 \pm 0.65$ & $6.28 \pm 1.09$ \\
\hline
\end{tabular}

Baseline body weight (BBW), final body weight (FBW), and body weight gain (BWG) in $\mathrm{ApoE}^{-/-}$ mice. Values are shown as the mean and standard error of the mean of each group $(n=4)$. Ctrl, control group. LPHs, lupine protein hydrolysates group. SD, standard group.

With these results, we can conclude that the effects observed in this study were not related to BWG of mice, which remained unchanged between the three experimental groups.

\subsection{LPHs Exert an Anxiolytic-Like Effect}

Representative images of the tracks of mice in the maze appear in the Figure 2A-C. WD mice spent significantly less time in opened arms and more in closed arms compared to the SD group. This effect was reversed after LPH supplementation (Figure 2D,E). Moreover, the time spent in the center was significantly lower in WD compared to SD and WD + LPHs (Figure 2F). Furthermore, other anxiety-related behaviors were evaluated. The number of head dips was significantly lower after WD intake compared to SD, but not in the WD + LPHs group (Figure 2G). There were no differences in the rears between groups (Figure 2H). 


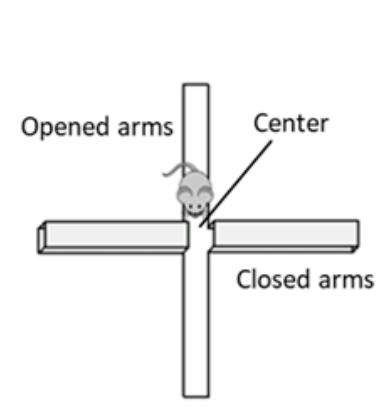

D

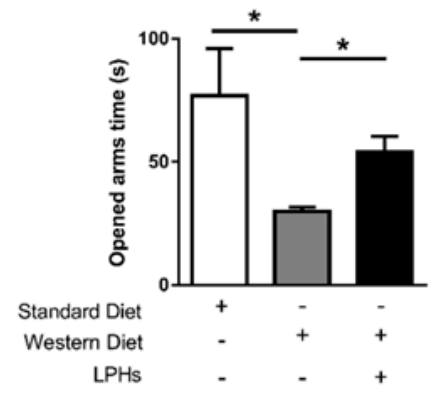

B

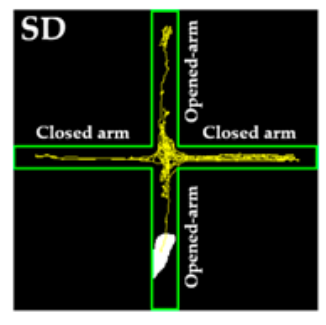

E

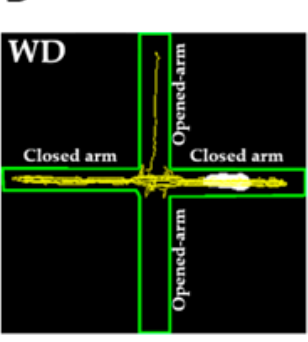

C

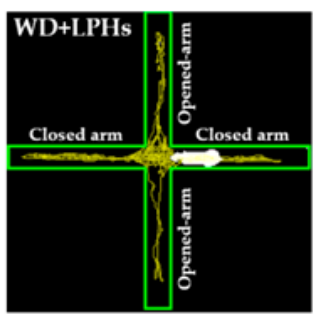

$\mathbf{F}$
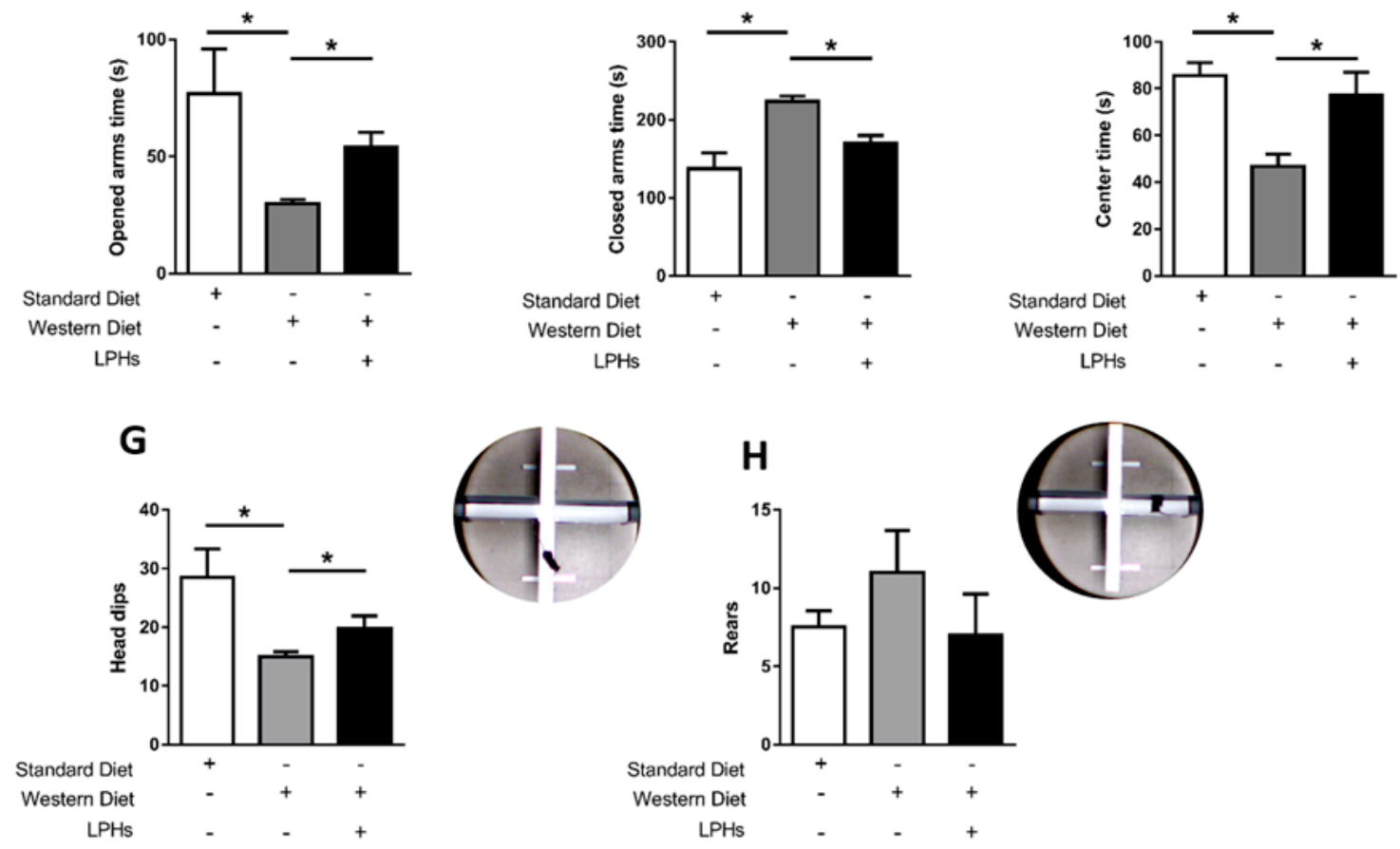

Figure 2. Effects of LPHs on WD-induced anxiety behaviors. Representative images of the tracks of mice in the maze (AC). Time spent in opened arms (D), closed arms (E), and center (F), plus head dips (G) and rears $(\mathbf{H})$ for the experimental groups. Values are shown as the mean and standard error of the mean of each group $(n=4)$.

It is well-known that WD accelerates the cognitive deficits in $\mathrm{ApoE}^{-/-}$mice [5]. In the present study, we observed that WD increased anxiety levels significantly. These results are in accordance with previous studies in humans [10] and mice [11]. LPHs, therefore, were shown to minimize the anxiolytic effects caused by the intake of the WD. It has already been shown that bioactive peptides from soy [6] may exert anti-anxiety activity, but, to the best of our knowledge, this is the first time that a Lupine hydrolysate showed anxiolytic-like properties.

\subsection{LPHs Do Not Alter Spatial Memory}

The latency time, which reflects the ability to learn the platform location, is shown in Figure 3B. During the training phase, we did not observe any significant differences between groups. This result revealed that all groups were equally capable of learning the task. The probe phase shows that there were no differences in the time spent in the platform zone (Figure 3C) among groups. Interestingly, a decreasing trend in the total distance traveled after WD consumption was observed $(p<0.058$, compared to SD), which was reverted after LPH treatment ( $p<0.0571$ compared to WD) (Figure 3D). 
A

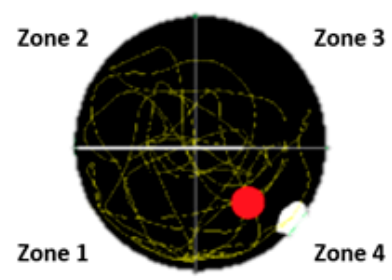

B

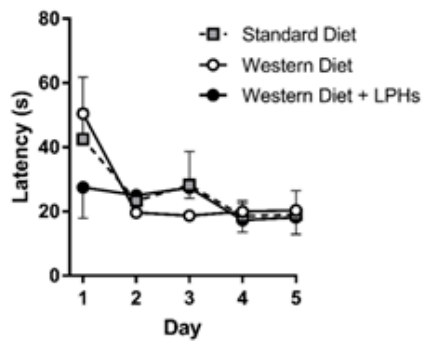

C

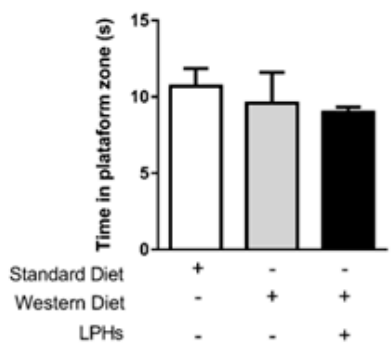

D

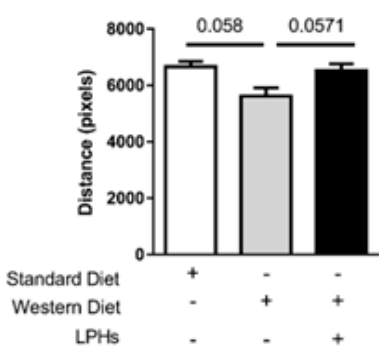

Figure 3. Effects of LPHs on WD-induced cognitive deficits. Representative image of the MWM (A), learning effects (B), time in the platform zone $(\mathbf{C})$, and distance traveled $(\mathbf{D})$ of each experimental group. Values are shown as the mean and standard error of the mean of each group $(n=4)$.

Our results revealed no impairment of memory or spatial learning after WD consumption. This fact could be associated with the age of the mice and time of WD consumption [9]. Unlike other peptides [12], LPHs improved neither spatial memory nor learning after a 14-week treatment.

\section{Conclusions}

This is the first study showing that an LPH treatment causes anxiolytic effects, pointing to LPHs as a potential component of future nutritional therapies in AD patients with anxiety, a serious side effect of this disease.

Institutional Review Board Statement: The experimental procedures were approved by the Virgen Macarena-Virgen del Rocío University Hospital ethical committee (reference number 21/06/2016/105) and conducted under Spanish legislation and the EU Directive 2010/63/EU for animal experiments.

Funding: This research was funded by the Spanish Government, Ministerio de Economía y Competitividad [AGL2012-40247-C02-01 and AGL2012-40247-C02-02], the Andalusian Government Ministry of Health [PC-0111-2016-0111], and the PAIDI Program from the Andalusian Government [CTS160]. G.S.-S. and I.C.-C. were supported by FPU grants from the Spanish Ministerio de Educación, Cultura y Deporte, [FPU16/02339] and [FPU13/01210], respectively. I.B. and E.P.E. were supported by the VI Program of Inner Initiative for Research and Transfer of University of Seville (VI PPIT-US).

\section{Informed Consent Statement: Not applicable}

Conflicts of Interest: The authors declare no conflict of interest.

\section{References}

1. Alzheimer's Association. Alzheimer's disease facts and figures. Alzheimers Dement 2010, 6, 158-194, doi:10.1002/alz.12068.

2. Rosenberg, P.B.; Mielke, M.M.; Appleby, B.; Oh, E.; Leoutsakos, J.M.; Lyketsos, C.G. Neuropsychiatric symptoms in MCI subtypes: The importance of executive dysfunction. Int. J. Geriatr. Psychiatry 2011, 26, 364-372, doi:10.1002/gps.2535.

3. Silva, M.V.F.; Loures, C.d.M.G.; Alves, L.C.V.; de Souza, L.C.; Borges, K.B.G.; das Graças Carvalho, M. Alzheimer's disease: Risk factors and potentially protective measures. J. Biomed. Sci. 2019, 26, 33, doi:10.1186/s12929-019-0524-y.

4. Kopp, W. How western diet and lifestyle drive the pandemic of obesity and civilization diseases. Diabetes Metab. Syndr. Obes. 2019, 12, 2221, doi:10.2147/DMSO.S216791.

5. Yuan, T.; Chu, C.; Shi, R.; Cui, T.; Zhang, X.; Zhao, Y.; Shi, X.; Hui, Y.; Pan, J.; Qian, R. ApoE-Dependent Protective Effects of Sesamol on High-Fat Diet-Induced Behavioral Disorders: Regulation of the Microbiome-Gut-Brain Axis. J. Agric. Food Chem. 2019, 67, 6190-6201, doi:10.1021/acs.jafc.9b01436.

6. Ohinata, K.; Agui, S.; Yoshikawa, M. Soymorphins, novel $\mu$ opioid peptides derived from soy $\beta$-conglycinin $\beta$-subunit, have anxiolytic activities. Biosci. Biotechnol. Biochem. 2007, 71, 2618-2621, doi:10.1271/bbb.70516. 
7. Cruz-Chamorro, I.; Álvarez-Sánchez, N.; del Carmen Millán-Linares, M.; del Mar Yust, M.; Pedroche, J.; Millán, F.; Lardone, P.J.; Carrera-Sánchez, C.; Guerrero, J.M.; Carrillo-Vico, A. Lupine protein hydrolysates decrease the inflammatory response and improve the oxidative status in human peripheral lymphocytes. Food Res. Int. 2019, 126, 108585, doi:10.1016/j.foodres.2019.108585.

8. Oda, A.; Kaneko, K.; Mizushige, T.; Lazarus, M.; Urade, Y.; Ohinata, K. Characterization of ovolin, an orally active tryptic peptide released from ovalbumin with anxiolytic-like activity. J. Neurochem. 2012, 122, 356-362, doi:10.1111/j.14714159.2012.07777.x.

9. Janssen, C.I.; Jansen, D.; Mutsaers, M.P.; Dederen, P.J.; Geenen, B.; Mulder, M.T.; Kiliaan, A.J. The effect of a high-fat diet on brain plasticity, inflammation and cognition in female ApoE4-knockin and ApoE-knockout mice. PLoS ONE 2016, 11, e0155307, doi:10.1371/journal.pone.0155307.

10. Puder, J.; Munsch, S. Psychological correlates of childhood obesity. Int. J. Obes. 2010, 34, S37-S43, doi:10.1038/ijo.2010.238.

11. Dutheil, S.; Ota, K.T.; Wohleb, E.S.; Rasmussen, K.; Duman, R.S. High-fat diet induced anxiety and anhedonia: Impact on brain homeostasis and inflammation. Neuropsychopharmacology 2016, 41, 1874-1887, doi:10.1038/npp.2015.357.

12. Xu, X.; Liang, R.; Li, D.; Jiang, C.; Lin, S. Evaluation of sea cucumber peptides-assisted memory activity and acetylation modification in hippocampus of test mice based on scopolamine-induced experimental animal model of memory disorder. J. Funct. Foods 2020, 68, 103909, doi:10.1016/j.jff.2020.103909. 